\title{
A Two-Component Generalization of the Integrable rdDym Equation ${ }^{\star}$
}

Oleg I. MOROZOV

Institute of Mathematics and Statistics, University of Tromsø, Tromsø 90-37, Norway

E-mail: Oleg.Morozov@uit.no

Received May 26, 2012, in final form August 09, 2012; Published online August 11, 2012

http://dx.doi.org/10.3842/SIGMA.2012.051

\begin{abstract}
We find a two-component generalization of the integrable case of rdDym equation. The reductions of this system include the general rdDym equation, the Boyer-Finley equation, and the deformed Boyer-Finley equation. Also we find a Bäcklund transformation between our generalization and Bodganov's two-component generalization of the universal hierarchy equation.
\end{abstract}

Key words: coverings of differential equations; Bäcklund transformations

2010 Mathematics Subject Classification: 35A30; 58H05; 58J70

\section{Introduction}

Recent papers $[3,8,16]$ provide two-component generalizations for the hyper-CR Einstein-Weil structure equation $[6,22]$

$$
s_{y y}=s_{t x}+s_{y} s_{x x}-s_{x} s_{x y}
$$

Plebański's second heavenly equation [25]

$$
s_{x z}=s_{t y}+s_{x x} s_{y y}-s_{x y}^{2}
$$

and the universal hierarchy equation $[18,19,22]$

$$
s_{x x}=s_{x} s_{t y}-s_{t} s_{x y} .
$$

Namely, equations (1.1)-(1.3) appear from systems

$$
\begin{aligned}
& s_{y y}=s_{t x}+\left(s_{y}+r\right) s_{x x}-s_{x} s_{x y}, \\
& r_{y y}=r_{t x}+\left(s_{y}+r\right) r_{x x}-s_{x} r_{x y}+r_{x}^{2} ; \\
& s_{x z}=s_{t y}+s_{x x} s_{y y}-s_{x y}^{2}+r, \\
& r_{x z}=r_{t y}+s_{y y} r_{x x}+s_{x x} r_{y y}-2 s_{x y} r_{x y},
\end{aligned}
$$

and

$$
\begin{gathered}
s_{x x}=e^{r}\left(s_{x} s_{t y}-s_{t} s_{x y}\right), \\
\left(e^{-r}\right)_{x x}=s_{x} r_{t y}-s_{t} r_{x y},
\end{gathered}
$$

respectively, by substituting for $r=0$. Other reductions for (1.4) are found in [7, 16]: when $u=0$, system (1.4) gives the Khokhlov-Zabolotskaya (or dispersionless Kadomtsev-Petviashvili) equation

$$
v_{y y}=v_{t x}+v v_{x x}+v_{x}^{2}
$$

${ }^{\star}$ This paper is a contribution to the Special Issue "Geometrical Methods in Mathematical Physics". The full collection is available at http://www.emis.de/journals/SIGMA/GMMP2012.html 
while substituting for $v=u_{x}$ in (1.4) produces the normal form

$$
u_{y y}=u_{t x}+\left(u_{x}+u_{y}\right) u_{x x}-u_{x} u_{x y}
$$

for the family of equations studied in [7]. Also, we note the reduction $v=u_{y}$ for system (1.4). This reduction yields equation

$$
u_{y y}=u_{t x}-u_{x} u_{x y}
$$

studied in $[9,14,17,21]$.

As it was shown in [3], the reduction $s=x$ for system (1.6) gives the Boyer-Finley equation

$$
r_{t y}=\left(e^{-r}\right)_{x x} .
$$

The purpose of the present paper is to introduce the two-component generalization for equation

$$
u_{t y}=u_{x} u_{x y}-u_{y} u_{x x},
$$

which is integrable in the following sense: it has the differential covering $[2,11,12,13]$

$$
p_{t}=\left(u_{x}-\lambda\right) p_{x}, \quad p_{y}=\lambda^{-1} u_{y} p_{x}
$$

containing the non-removable parameter $\lambda \neq 0$ [20]. We show that reductions of the generalization include the general $r$-th dispersionless Dym equation [1]

$$
u_{t y}=u_{x} u_{x y}+\kappa u_{y} u_{x x}
$$

the Boyer-Finley equation (1.7), and the deformed Boyer-Finley equation. Also we find a Bäcklund transformation between our generalization and Bodganov's two-component generalization (1.6) of the universal hierarchy equation (1.3).

\section{The two-component generalization}

Along with the covering (1.9) equation (1.8) has the covering

$$
q_{t}=\left(u_{x}-q\right) q_{x}, \quad q_{y}=u_{y} q^{-1} q_{x},
$$

which can be obtained by the method of [20]. While the coverings (1.9) and (2.1) are not equivalent w.r.t. the pseudo-group of contact transformations, (2.1) can be derived from (1.9) by the following procedure, see, e.g., [24]. We consider the function $p=p(t, x, y)$ from (1.9) to be defined implicitly by the equation $q(t, x, y, p(t, x, y))=\lambda$ with $q_{p} \neq 0$. Then for $\left(x^{1}, x^{2}, x^{3}\right)=$ $(t, x, y)$ we have $q_{x^{i}}+q_{p} p_{x^{i}}=0$, so $p_{x^{i}}=-q_{x^{i}} / q_{p}$. Substituting these into (1.9) yields (2.1).

Our main observation in this paper is that the covering (2.1) allows the generalization

$$
q_{t}=\left(u_{x}-q+v\right) q_{x}+v_{x} q, \quad q_{y}=u_{y} q^{-1} q_{x}+v_{y} .
$$

This system is compatible whenever the two-component system

$$
\begin{aligned}
& u_{t y}=\left(u_{x}+v\right) u_{x y}-u_{y} u_{x x}, \\
& v_{t y}=\left(u_{x}+v\right) v_{x y}-u_{y} v_{x x}+v_{x} v_{y}
\end{aligned}
$$

holds. In other words, (2.2) is a covering for system (2.3), (2.4). 


\section{Reductions}

By the construction, we have the following reduction for system (2.2):

Reduction A. Substituting for $v=0$ in equations (2.3), (2.2) gives equations (1.8) and (2.1), while (2.4) becomes an identity.

Also, we have three other reductions.

Reduction B. If we put $v=-\left(\kappa^{-1}+1\right) u_{x}$, then $(2.3)$ gets the form

$$
u_{t y}=-\kappa^{-1} u_{x} u_{x y}-u_{y} u_{x x}
$$

while (2.4) is its differential consequence. The transformation $u \mapsto-\kappa u$ maps (3.1) to (1.10). The corresponding reduction of (2.2) produces the covering of (1.10) studied in [20,23].

Reduction $C$. Taking $v=-u_{x}$ in (2.3), (2.4), we obtain

$$
u_{t y}=-u_{y} u_{x x}
$$

and its differential consequence. Then we divide this equation by $u_{y}$, differentiate w.r.t. $y$ and put $u_{y}=-e^{w}$. This gives the Boyer-Finley equation [4]

$$
w_{t y}=\left(e^{w}\right)_{x x}
$$

This equation is equation (1.7) in a different notation. Substituting for $q=e^{p}$ in the corresponding reduction of $(2.2)$, we have the covering $[10,15,26]$ for equation $(3.2)$ :

$$
p_{t}=w_{t}-e^{p} p_{x}, \quad p_{y}=e^{w-p}\left(w_{x}-p_{x}\right) .
$$

Reduction D. Finally, when we put $v=u_{y}-u_{x}$ into (2.3) and (2.4), we get the equation

$$
u_{t y}=u_{y}\left(u_{x y}-u_{x x}\right)
$$

and its differential consequence. Then for $u_{y}=e^{w}$ we have the deformed Boyer-Finley equation [5]

$$
w_{t y}=\left(e^{w}\right)_{x y}-\left(e^{w}\right)_{x x},
$$

and the corresponding reduction of equations (2.2) with $q=e^{s}$ gives the covering

$$
s_{t}=\left(e^{s}-e^{w}\right) s_{x}-w_{t}, \quad s_{y}=e^{w}\left(s_{x}-w_{x}+w_{y}\right) .
$$

for (3.3). This covering in other notations was found in $[5,20]$.

\section{Bäcklund transformations}

The substitution

$$
u_{x}=-v+\frac{s_{t}}{s_{x}}, \quad u_{y}=-\frac{e^{-r}}{s_{x}}, \quad v_{x}=\frac{r_{x} s_{t}}{s_{x}}-r_{t}, \quad v_{y}=-\frac{e^{-r} r_{x}}{s_{x}}
$$

maps system (2.2) to system

$$
q_{t}=\left(\frac{s_{t}}{s_{x}}-q\right) q_{x}+\left(\frac{s_{t} r_{x}}{s_{x}}-r_{t}\right) q, \quad q_{y}=-\frac{e^{-r}}{q s_{x}}\left(q_{x}+r_{x} q\right)
$$

found in [3]. This system is the two-component generalization of the covering

$$
q_{t}=\left(\frac{s_{t}}{s_{x}}-q\right) q_{x}, \quad q_{y}=-\frac{q_{x}}{q s_{x}} .
$$


of equation (1.3). The compatibility conditions for (4.2) coincide with (1.6). Solving (4.1) for $s_{t}, s_{x}, r_{t}, r_{x}$ yields

$$
s_{t}=-\left(u_{x}+v\right) \frac{e^{-r}}{u_{y}}, \quad s_{x}=-\frac{e^{-r}}{u_{y}}, \quad r_{t}=\frac{v_{y}}{u_{y}}, \quad r_{x}=\frac{\left(u_{x}+v\right) v_{y}}{u_{y}}-v_{x} .
$$

This system is compatible whenever equations (2.3), (2.4) are satisfied. Thus equations (4.1) define a Bäcklund transformation from (2.3), (2.4) to (1.6) with the inverse transformation (4.3). In particular, when $v=0$ and $r=0$, we have a Bäcklund transformation

$$
u_{x}=\frac{s_{t}}{s_{x}}, \quad u_{y}=-\frac{1}{s_{x}}
$$

between (1.1) and (1.3) with the inverse transformation

$$
s_{t}=-\frac{u_{x}}{u_{y}}, \quad s_{x}=-\frac{1}{u_{y}}
$$

\section{Acknowledgments}

I am very grateful to M.V. Pavlov and A.G. Sergyeyev for the valuable discussions. Also I'd like to thank M. Marvan and A.G. Sergyeyev for the warm hospitality in Mathematical Institute, Silezian University at Opava, Czech Republic, where this work was initiated and partially supported by the ESF project CZ.1.07/2.3.00/20.0002.

\section{References}

[1] Błaszak M., Classical R-matrices on Poisson algebras and related dispersionless systems, Phys. Lett. A 297 (2002), 191-195.

[2] Bocharov A.V., Chetverikov V.N., Duzhin S.V., Khor'kova N.G., Krasil'shchik I.S., Samokhin A.V., Torkhov Y.N., Verbovetsky A.M., Vinogradov A.M., Symmetries and conservation laws for differential equations of mathematical physics, Translations of Mathematical Monographs, Vol. 182, American Mathematical Society, Providence, RI, 1999.

[3] Bogdanov L.V., Non-Hamiltonian generalizations of the dispersionless 2DTL hierarchy, J. Phys. A: Math. Theor. 43 (2010), 434008, 8 pages, arXiv:1003.0287.

[4] Boyer C.P., Finley III J.D., Killing vectors in self-dual, Euclidean Einstein spaces, J. Math. Phys. 23 (1982), 1126-1130.

[5] Dryuma V.S., Non-linear multi-dimensional equations related to commuting vector fields, in Book of abstracts of International Conference "Differential Equations and Related Topics" dedicated to I.G. Petrovskii, XXII Joint Session of Moscow Mathematical Society and Petrovskii Seminar (Moscow, May 21-26, 2007), Moscow Lomonosov State University, Moscow, 2007, 78.

[6] Dunajski M., A class of Einstein-Weyl spaces associated to an integrable system of hydrodynamic type, J. Geom. Phys. 51 (2004), 126-137, nlin.SI/0311024.

[7] Dunajski M., An interpolating dispersionless integrable system, J. Phys. A: Math. Theor. 41 (2008), 315202, 9 pages, arXiv:0804.1234.

[8] Dunajski M., Anti-self-dual four-manifolds with a parallel real spinor, Proc. R. Soc. Lond. Ser. A 458 (2002), 1205-1222, math.DG/0102225.

[9] Ferapontov E.V., Moro A., Sokolov V.V., Hamiltonian systems of hydrodynamic type in $2+1$ dimensions, Comm. Math. Phys. 285 (2009), 31-65, arXiv:0710.2012.

[10] Kashaev R.M., Saveliev M.V., Savelieva S.A., Vershik A.M., On nonlinear equations associated with Lie algebras of diffeomorphism groups of two-dimensional manifolds, in Ideas and Methods in Mathematical Analysis, Stochastics, and Applications (Oslo, 1988), Cambridge University Press, Cambridge, 1992, 295307. 
[11] Krasil'shchik I.S., Lychagin V.V., Vinogradov A.M., Geometry of jet spaces and nonlinear partial differential equations, Advanced Studies in Contemporary Mathematics, Vol. 1, Gordon and Breach Science Publishers, New York, 1986.

[12] Krasil'shchik I.S., Vinogradov A.M., Nonlocal symmetries and the theory of coverings: an addendum to Vinogradov's "Local symmetries and conservation laws", Acta Appl. Math. 2 (1984), 79-96.

[13] Krasil'shchik I.S., Vinogradov A.M., Nonlocal trends in the geometry of differential equations: symmetries, conservation laws, and Bäcklund transformations, Acta Appl. Math. 15 (1989), 161-209.

[14] Krichever I.M., The $\tau$-function of the universal Whitham hierarchy, matrix models and topological field theories, Comm. Pure Appl. Math. 47 (1994), 437-475, hep-th/9205110.

[15] Malykh A.A., Nutku Y., Sheftel M.B., Winternitz P., Invariant solutions of complex Monge-Ampère equation and gravitational instantons, Phys. Atomic Nuclei 61 (1989), 1986-1989.

[16] Manakov S.V., Santini P.M., Cauchy problem on the plane for the dispersionless Kadomtsev-Petviashvili equation, JETP Lett. 83 (2006), 462-466, nlin.SI/0604023.

[17] Mañas M., Medina E., Martínez Alonso L., On the Whitham hierarchy: dressing scheme, string equations and additional symmetries, J. Phys. A: Math. Gen. 39 (2006), 2349-2381, nlin.SI/0509017.

[18] Martínez Alonso L., Shabat A.B., Energy-dependent potentials revisited: a universal hierarchy of hydrodynamic type, Phys. Lett. A 299 (2002), 359-365, nlin.SI/0202008.

[19] Martínez Alonso L., Shabat A.B., Hydrodynamic reductions and solutions of the universal hierarchy, Theoret. and Math. Phys. 140 (2004), 1073-1085, nlin.SI/0312043.

[20] Morozov O.I., Contact integrable extensions of symmetry pseudo-groups and coverings of $(2+1)$ dispersionless integrable equations, J. Geom. Phys. 59 (2009), 1461-1475, arXiv:0809.1218.

[21] Pavlov M.V., Classification of integrable Egorov hydrodynamic chains, Theoret. and Math. Phys. 138 (2004), 45-58, nlin.SI/0603055.

[22] Pavlov M.V., Integrable hydrodynamic chains, J. Math. Phys. 44 (2003), 4134-4156, nlin.SI/0301010.

[23] Pavlov M.V., The Kupershmidt hydrodynamic chains and lattices, Int. Math. Res. Not. (2006), Art. ID 46987, 43 pages, nlin.SI/0604049.

[24] Pavlov M.V., Chang J.H., Chen Y.T., Integrability of the Manakov-Santini hierarchy, arXiv:0910.2400.

[25] Plebański J.F., Some solutions of complex Einstein equations, J. Math. Phys. 16 (1975), 2395-2402.

[26] Zakharov V.E., Integrable systems in multidimensional spaces, in Mathematical Problems in Theoretical Physics (Berlin, 1981), Lecture Notes in Phys., Vol. 153, Springer, Berlin, 1982, 190-216. 